Artículo original

\title{
Síndrome del lacertus fibrosus
}

\author{
Lacertus fibrosus syndrome
}

\author{
Roberto Meléndez ${ }^{1,}$ Gonzalo Martínez², Natalia Salamanca ${ }^{3}$ \\ ${ }^{1}$ Clínica Reina Sofía, Cirugía de Mano, Bogotá D.C., Colombia. \\ ${ }^{2}$ Universidad del Rosario, Especialización en Ortopedía y Traumatología, Bogotá D.C., Colombia. \\ ${ }^{3}$ Unidad Médica CECIMIN, Bogotá D.C., Colombia. \\ Correspondencia: Roberto Meléndez. Clínica Reina Sofía. Bogotá D.C. Colombia \\ Correo electrónico cromeles@gmail.com.
}

\section{Información del artículo Resumen}

Recibido: 09/11/2020 El lacertus fibrosus del bíceps o expansión aponeurótica del bíceps es una estructura a la Aceptado: 18/03/2021 que suele prestársele poca atención frente a otras unidades miotendinosas de destacada importancia como el bíceps brachii o el tríceps. Por esta razón, en general es escaso el conocimiento que se tiene de ella respecto a su anatomía, biomecánica, fisiología y, sobre

Palabras clave: Anatomía; Nervio mediano; Neuropatía mediana.

Keywords: Anatomy; Median nerve; Median todo, patología; en las descripciones del síndrome del pronador redondo se le menciona de manera incidental. neuropathy.

DOI: https://doi.org/10.25214/28056272.1179

El presente es un estudio descriptivo observacional de tipo retrospectivo hecho con el propósito de revisar la anatomía del lacertus fibrosus, plantear aspectos de su biomecánica y presentar la revisión de una serie de 29 casos identificados a partir de 4100 historias clínicas realizadas entre el 13 de enero de 2015 y el 12 de febrero de 2020.

En la revisión de la literatura hecha para la presente investigación se encontró que dicha patología predominó en las mujeres y en los mayores de 56 años, siendo el principal motivo de consulta el dolor en la cara antero-medial del brazo distal y del codo. Además, se halló que su presentación clínica con frecuencia se acompañaba de los síndromes del pronador redondo y del interóseo posterior.
Abstract
The lacertus fibrosus of the biceps brachii or aponeurotic expansion of the biceps is a structure to which little attention is generally paid compared to other myotendinous units of outstanding importance such as the biceps or the triceps. For this reason, in general, little is known about it in regard to its anatomy, biomechanics, physiology, and much less in terms of her pathology. In descriptions of pronator teres syndrome, it is incidentally mentioned. This is a retrospective descriptive observational study with the purpose of reviewing its related anatomy, outline some biomechanical aspects and present the re- view of a series of 29 cases identified from 4100 medical records in a 5 year period from January 132015 to February 12 2020; during that period of time, we found the disease to affect more frequent women over 56 years of age, being the main reason for consultation pain on the anteromedial aspect of the proximal forearm and being frequently associated with pronator teres and posterior interosseus syndromes. 


\section{Introducción}

Clásicamente denominada como lacertus fibrosus (del latín: lagarto) o expansión aponeurótica del bíceps, en la actualidad a esta estructura también se le conoce como aponeurosis musculi bicipitis brachii, bicipital fascia, Pirogoff aponeurosis, semilunar fascia o "grace Dieu" fascia".

\section{Anatomía}

El lacertus fibrosus se describe como una lámina aponeurótica que se origina en el lado interno del tendón distal del bíceps brachii, se expande en forma triangular en sentido medial y distal a modo de abanico y cruza en sentido oblicuo la cara anterior de los músculos epitrocleares para insertarse en la fascia antebraquial y en el borde dorsal del cúbito ${ }^{1-4}$.

Esta es una estructura que varía de aspecto de un paciente a otro: en ocasiones se la encuentra muy bien caracterizada (Figura 1) al ser ancha, gruesa y firme, pero en otras es laxa y angosta. Con frecuencia es de forma triangular, pero en ocasiones es rectangular o trapezoidal ${ }^{2}$.

Figura 1. Aspectos del lacertus fibrosus en su anatomía de superficie y quirúrgica.

Fuente: Documento obtenido durante la elaboración del estudio.
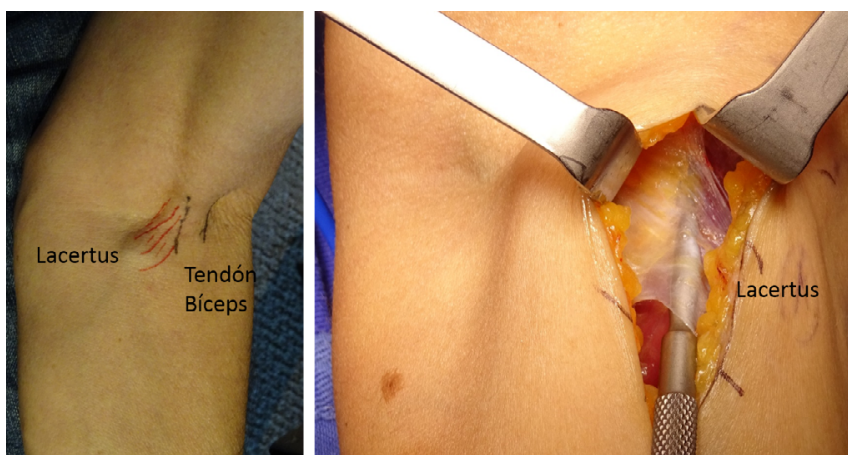

Por otra parte, Snoeck et al..$^{5}$ confirmaron en estudios cadavéricos la existencia de una capa profunda antes descrita por Eames et al. ${ }^{6}$, la cual se inserta en el borde antero-medial del cúbito rodeando la cara lateral del pronador redondo (Figura 2).

Figura 2. Capas superficial y profunda del lacertus fibrosus. Fuente: Elaboración propia.

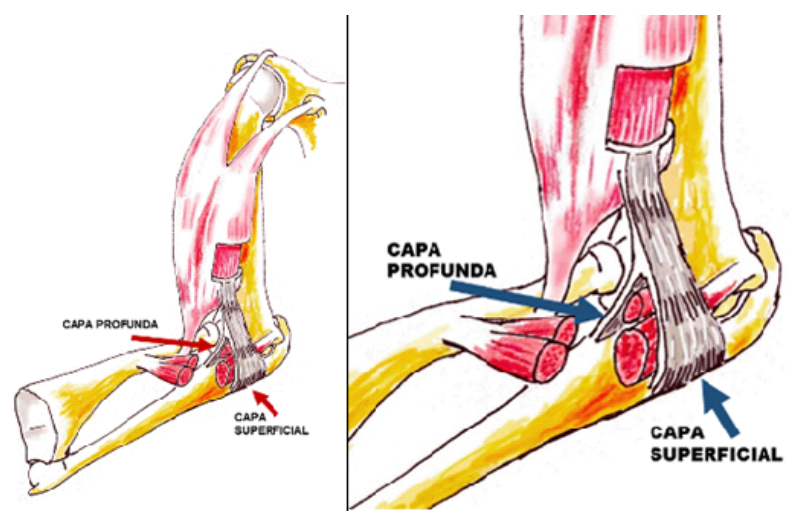

\section{Biomecánica}

El lacertus fibrosus es una estructura influenciada por diversos factores mecánicos: la flexión y extensión del codo, la pronación o supinación del antebrazo y la extensión o flexión de la muñeca; además, esta influencia es diferente si cada uno de dichos movimientos se realiza en forma pasiva o activa. Este complejo funcional plantea, entonces, muchas combinaciones de movimientos y su análisis es uno de los objetivos del presente trabajo.

Es de anotar que, en principio, el bíceps es considerado un músculo biarticular, tal como lo describe Kapandji ${ }^{7}$, pues sobrepasa en la parte proximal a la articulación gleno-humeral y en la distal a la braqui-antebraquial. Sin embargo, se debe tener en cuenta que tanto en su extremo proximal como distal los tendones de este se dividen en dos, conformando un músculo de cuatro tendones: proximalmente en la porción corta que se inserta en la apófisis coracoides y la larga que se inserta en el tubérculo supraglenoideo, y distalmente en el "tendón del bíceps" que se inserta en la tuberosidad bicipital del radio (considerado como la inserción principal) 4 y en el lacertus fibrosus que se inserta en la aponeurosis flexopronadora y en el borde posterior del cúbito, considerado como una inserción secundaria ${ }^{4}$ (Figura 3). Es importante tener presente que algunos autores han descrito que la porción corta del bíceps da origen al lacertus fibrosus y a una parte del tendón del bíceps, mientras que la porción larga únicamente contribuye a la formación del tendón principal².

Figura 3. Esquema del bíceps como músculo de cuatro tendones. Fuente: Elaboración propia.

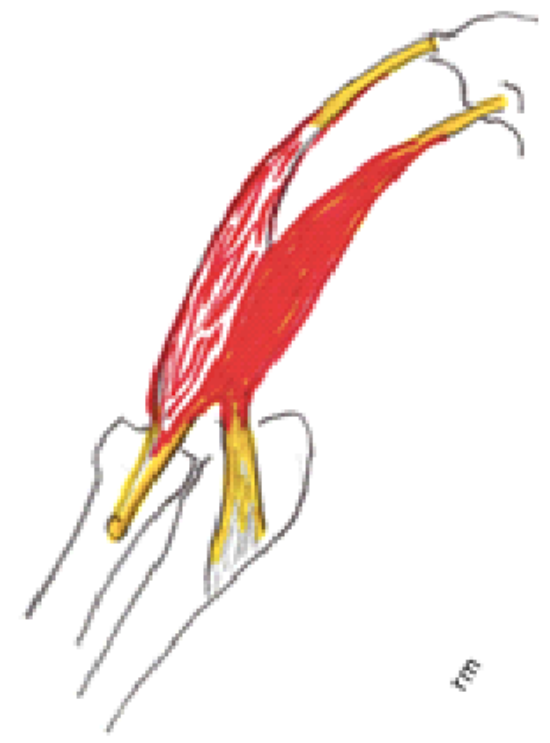

Teniendo en cuenta estas dos características, desde el punto de vista de la función se considera que en ambos extremos se produce un efecto de división de la fuerza de tracción que divide la acción del músculo proximalmente en puntos separados de un solo hueso (el omoplato) y distalmente en dos huesos (el radio y el cúbito) a su vez en relación compleja mediante la articulación entre el radio y el cúbito (articulación radio-cubital proximal, espacio interóseo y articulación radio-cubital distal). En este sentido, al bíceps se le debe considerar como un músculo triarticular (Figura 4). 
Figura 4. Concepto del bíceps como músculo triarticular. Fuente: Elaboración propia.

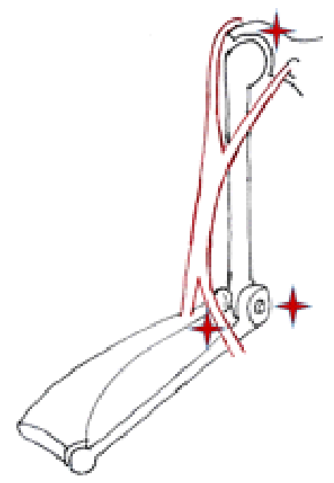

El objetivo del presente estudio es analizar los cambios de relajación/tensión que ocurren en el tendón del bíceps y en el lacertus fibrosus durante los movimientos de flexión/extensión del codo, pronación/supinación del antebrazo y flexión/extensión de la muñeca. Dado que se trata de una estructura triarticular y de varios momentos posicionales, se genera un gran número de combinaciones, las cuales se analizan a continuación y se presentan en la Tabla 1. El análisis se limita a estos movimientos ya que si se incluyera la influencia de los movimientos del hombro y el puño, el número de combinaciones sería mucho mayor y el análisis muy complejo. Es pertinente aclarar que el presente es un estudio preliminar, hipotético y original sobre aspectos sin antecedentes en la literatura y que probablemente constituye un punto de partida para una mejor comprensión de la biomecánica del lacertus fibosus y del bíceps brachii.

Tabla 1. Cambios de relajación/tensión en el tendón del bíceps y en el lacertus fibrosus en diversas posiciones del codo, el antebrazo y la muñeca.

\section{Posiciones}

\section{Codo en extensión}

\section{Bíceps relajado parcialmente}

Lacertus fibrosus tensionado parcialmente y trasladado en sentido proximal$$
\text { dento }
$$

Antebrazo en supinación pasiva

Antebrazo en pronación pasiva
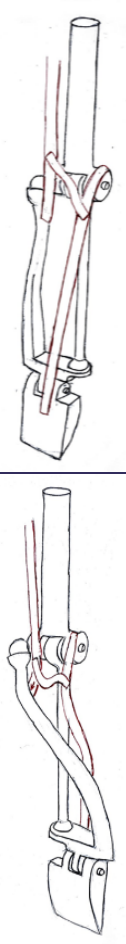

Bíceps estirado totalmente

Lacertus fibrosus relajado totalmente y trasladado en sentido distal

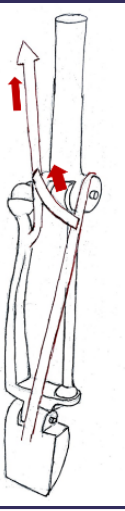

Bíceps contraído parcialmente

Lacertus fibrosus tensionado parcialmente y trasladado en sentido proximal 


\section{Codo en extensión}

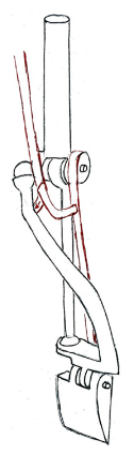

\section{Bíceps estirado totalmente}

Lacertus fibrosus relajado totalmente y trasladado en sentido distal

\section{Codo en flexión pasiva}

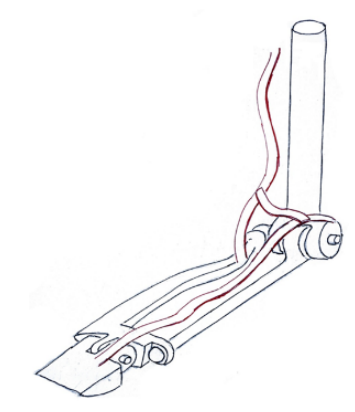

Bíceps relajado totalmente

Lacertus fibrosus relajado parcialmente y trasladado en sentido proximal

6

Antebrazo en pronación pasiva

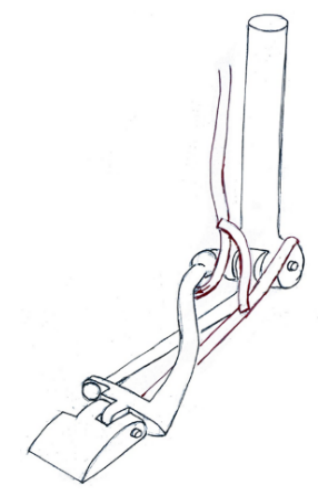

Bíceps estirado parcialmente

Lacertus fibrosus relajado totalmente y trasladado en sentido distal

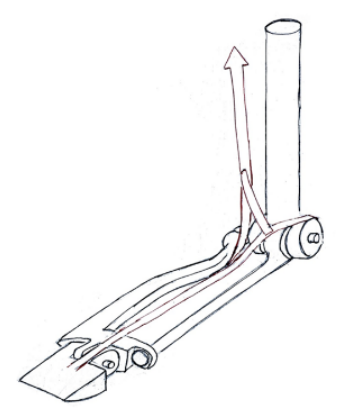

Bíceps contraído parcialmente

Lacertus fibrosus tensionado parcialmente y trasladado en sentido proximal 


\section{Codo en extensión}

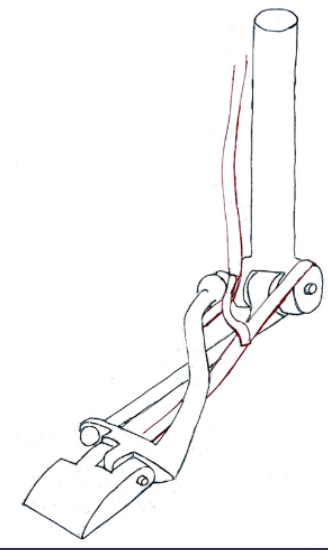

Bíceps estirado parcialmente

Lacertus fibrosus relajado totalmente y trasladado en sentido distal

\section{Codo en flexión activa}

9

Antebrazo en supinación pasiva

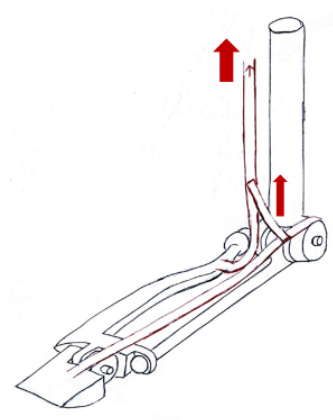

Bíceps contraído parcialmente

Lacertus fibrosus tensionado parcialmente y trasladado en sentido proximal

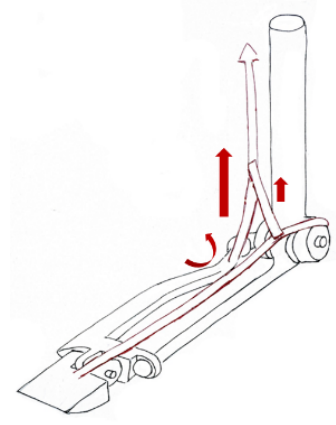

Bíceps contraído totalmente

Lacertus fibrosus tensionado parcialmente y trasladado en sentido proximal

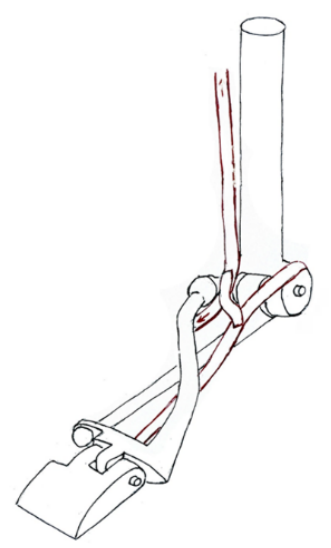

Bíceps estirado parcialmente

Lacertus fibrosus relajado totalmente y trasladado en sentido distal 
Lacertus fibrosus relajado totalmente y trasladado en sentido distal

Fuente: Elaboración propia.

\section{Cambios adicionales en la masa flexopronadora y en el lacertus fibrosus}

Los cambios en la masa flexopronadora ocurren según la posición de la muñeca: si está en flexión pasiva, la masa se relaja; si está en extensión pasiva, la masa se estira; si está en flexión activa, la masa se tensiona, y si está en extensión activa, la masa se estira.

Según los autores del presente estudio, en el lacertus fibrosus ocurren cambios que podrían definirse como un efecto limpiaparabrisas: al ser esta estructura de forma triangular o trapezoidal, en su desplazamiento distal se tensiona su parte proximal y se relaja la distal, mientras que en su desplazamiento proximal se tensiona su parte distal y se relaja la proximal (Figura 5).

Figura 5. Efecto limpiaparabrisas en el lacertus fibrosus. Fuente: Elaboración propia.

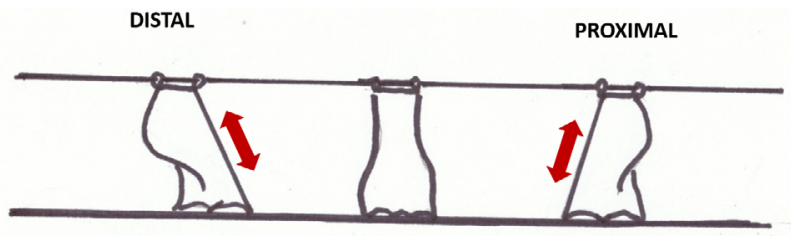

Por otra parte, es posible deducir que los cambios mencionados se traducen en un efecto de fuerza transversa (cizallamiento): si el lacertus fibrosus está relajado, permite el desplazamiento lateral del tendón del bíceps, aunque no más allá del eje de la unidad miotendínea, y si está tensionado, ejerce una fuerza de tracción medial sobre el tendón del bíceps (Figura 6).

Figura 6. Efecto de fuerza transversa ejercida por el lacertus fibrosus.

Fuente: Elaboración propia.

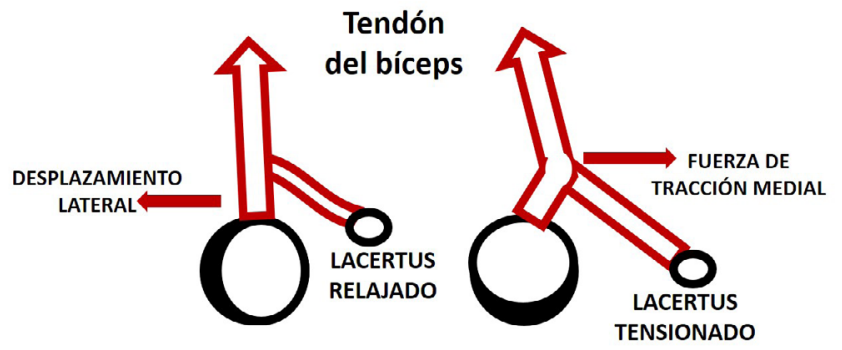

\section{Funciones del lacertus fibrosus}

1. De protección: le da protección anatómica a la arteria braquial y al nervio mediano, además sirve como un anclaje anatómico adicional del tendón del bíceps, refuerza la fascia antebraquial, alivia al radio de parte de la fuerza generada por el tendón principal y tracciona el borde posterior del cúbito medialmente para facilitar la supinación del antebrazo.

2. De propiocepción: provee información propioceptiva al bíceps basada en la actividad muscular del antebrazo.

3. Mecánicas: incrementa la tensión del tendón principal, dirige al tendón del bíceps en sentido medial durante su proceso de desenrollamiento para favorecer el sistema triarticular de la supinación ${ }^{1,2,5,8}$ y funciona como flexor del codo en presencia de una ruptura o arrancamiento del tendón principal del bíceps ${ }^{8}$ (Figura 7).

Figura 7. Lacertus fibrosus actuando como flexor del codo. Fuente: Elaboración propia.
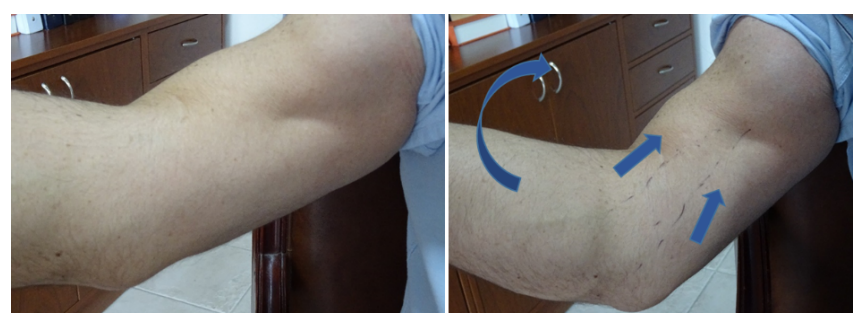

\section{Patología}

Spinner ${ }^{9}$ considera que el lacertus fibrosus es una estructura que puede causar compresión del nervio mediano, por lo que describe una maniobra diagnóstica que consiste en realizar simultáneamente la supinación del antebrazo y la flexión del codo contra resistencia para desencadenar el síntoma de dolor.

Otros autores incluyen al lacertus fibrosus como una de las estructuras que pueden causar compresión del nervio mediano y de la arteria braquial dentro del síndrome del pronador redondo $0^{2,10,11}$. Es importante mencionar que se considera que este último nombre es inadecuado por hacer referencia únicamente al músculo pronador redondo, siendo que son varias las estructuras que pueden causar compresión del nervio en la cara antero-medial del codo: el ligamento de 
Struthers, el lacertus fibrosus, la arcada del pronator teres y la arcada del flexor digitorum superficialis.

Hasta aquí es claro que las estructuras mencionadas pueden llevar a la compresión del nervio mediano; sin embargo, se hace énfasis en que una vez hecho el diagnóstico sindromático, el clínico debe buscar la causa por la cual estas estructuras que son normales pueden generar un efecto compresivo para poder hacer un diagnóstico etiológico.

Entre las posibles causas están: edema de origen hormonal (hipotiroidismo, embarazo, lactancia o uso de anticonceptivos), edema de otras causas (cardiopatías, neuropatías, desnutrición o linfedema), retracciones fasciales o miotendíneas por mala postura, procesos inflamatorios postraumáticos (contusión, sobreuso o sobreesfuerzo), enfermedad inflamatoria sistémica (enfermedad reumática o lupus), hipertrofia muscular secundaria a ejercicio físico, cambios en la estructura osteoarticular (fracturas consolidadas en posición viciosa, luxaciones o luxofracturas), cambios postraumáticos (fibrosis o retracciones compartimentales), cambios secundarios a enfermedades metabólicas (gota), etc. ${ }^{10}$.

La hipertrofia muscular propia de los atletas también es considerada como causa de compresión del nervio mediano por algunos autores, quienes recomiendan seccionar el lacertus fibrosus y liberar las otras estructuras mencionadas ${ }^{12,13}$. Anteriormente se ha sugerido que la denominación apropiada para las compresiones del nervio mediano por encima del codo debería ser "neuropatía compresiva proximal del nervio mediano" y que la expresión síndrome del pronador redondo se debería reservar para las compresiones ocurridas exclusivamente en dicho músculo².

Teniendo en cuenta que en la práctica clínica se han identificado casos con queja de dolor localizado en la cara antero-medial del brazo distal y del antebrazo proximal, sin afectación epitroclear y sin signos de compresión del nervio mediano (casos que sugieren la existencia de una patología propia del lacertus fibrosus), se propone agruparlos bajo la denominación de síndrome del lacertus fibrosus.

\section{Aspectos clínicos}

Cuando el motivo de consulta es síndrome del lacertus fibrosus la queja principal es dolor en la cara antero-medial del tercio distal del brazo y del codo. Se pueden presentar cuadros agudos, de menos de 3 semanas de evolución, o crónicos. En los primeros el paciente suele referir dolor intenso, en ocasiones lacerante, localizado y sin manifestaciones distales (Figura 8), y en los segundos los síntomas suelen ser similares, con variaciones horarias, empeoramiento con el reposo de la noche y acentuación con las actividades manuales.

Figura 8. Dolor lacerante en el área del lacertus fibrosus. Fuente: Documento obtenido durante la realización del estudio.

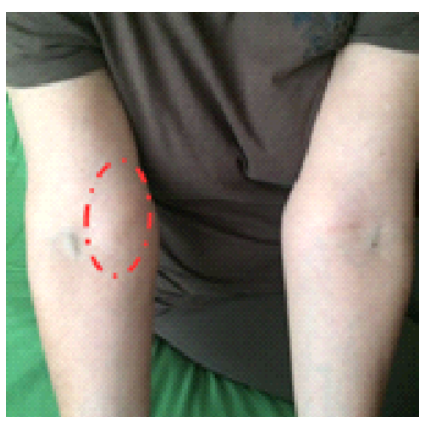

Durante la consulta, es indispensable establecer, mediante un interrogatorio detallado, un diagnóstico etiológico y precisar el tiempo de evolución para definir el tiempo de cronicidad; igualmente, se deben identificar los posibles factores desencadenantes o la presencia de factores predisponentes ${ }^{10}$. Entre los factores desencadenantes más frecuentes están los relacionados con sobreuso y/o sobreesfuerzo, como el caso de un tenista con dicho cuadro, de tres meses de evolución, en el cual el propio paciente relacionaba el origen de la moelstia con el hecho de haber cambiado la raqueta, el diámetro del agarre y la tensión de las cuerdas en su juego habitual. Por su parte, entre los factores predisponentes se deben considerar el hipotiroidismo, la enfermedad reumática, el embarazo, la lactancia y la gota. La hipertrofia muscular puede también conducir a la compresión del nervio mediano ${ }^{12,13}$.

Se recomienda iniciar el examen físico con una palpación superficial, suave, en toda el área, con el codo en flexión y continuarla realizando una extensión progresiva y lenta del codo. Esta fase puede ir seguida de la aplicación de presión digital siguiendo la anatomía del lacertus fibrosus, primero por su borde proximal, luego por el centro y después por el borde distal. Además, se sugiere realizar una percusión o una presión puntual digital suave en el trayecto del nervio mediano en busca de la aparición de un signo de Tinel (por presión o por percusión), así como realizar las maniobras correspondientes para identificar el dolor en el área de la arcada del pronador redondo.

Evaluados estos aspectos, se debe proceder a realizar maniobras de provocación, con las cuales se busca desencadenar dolor. Al respecto, Spinner ${ }^{9}$ recomienda hacer una maniobra diseñada con el fin de identificar si ocurre una compresión del nervio mediano por parte del lacertus fibrosus, y que consiste en efectuar la supinación del antebrazo con flexión del codo contra resistencia (Figura 9); por su parte, Eversmann ${ }^{11}$ recomienda flexionar de manera activa el codo con el antebrazo en pronación contra resistencia y flexión de la muñeca.

Figura 9. Maniobra de Spinner para verificar la compresión del nervio mediano por parte del lacertus Fuente: Elaboración con base en Spinner ${ }^{9}$.

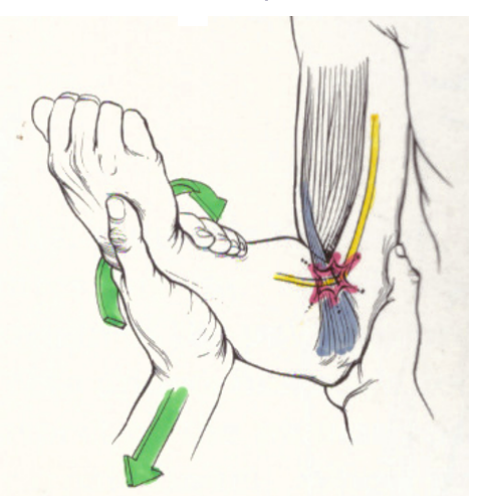

Siguiendo el objetivo de esta investigación, que va dirigida en primer lugar a valorar aspectos patológicos del lacertus fibrosus, entre las 12 posibilidades de combinación de posiciones analizadas en la Tabla 1 se seleccionaron 3 para ser aplicadas como maniobras de provocación siguiendo el criterio de colocar el codo y el antebrazo en las posiciones de máxima elongación y de máxima tensión del lacertus fibrosus (Tabla 2). 
Tabla 2. Maniobras de provocación.

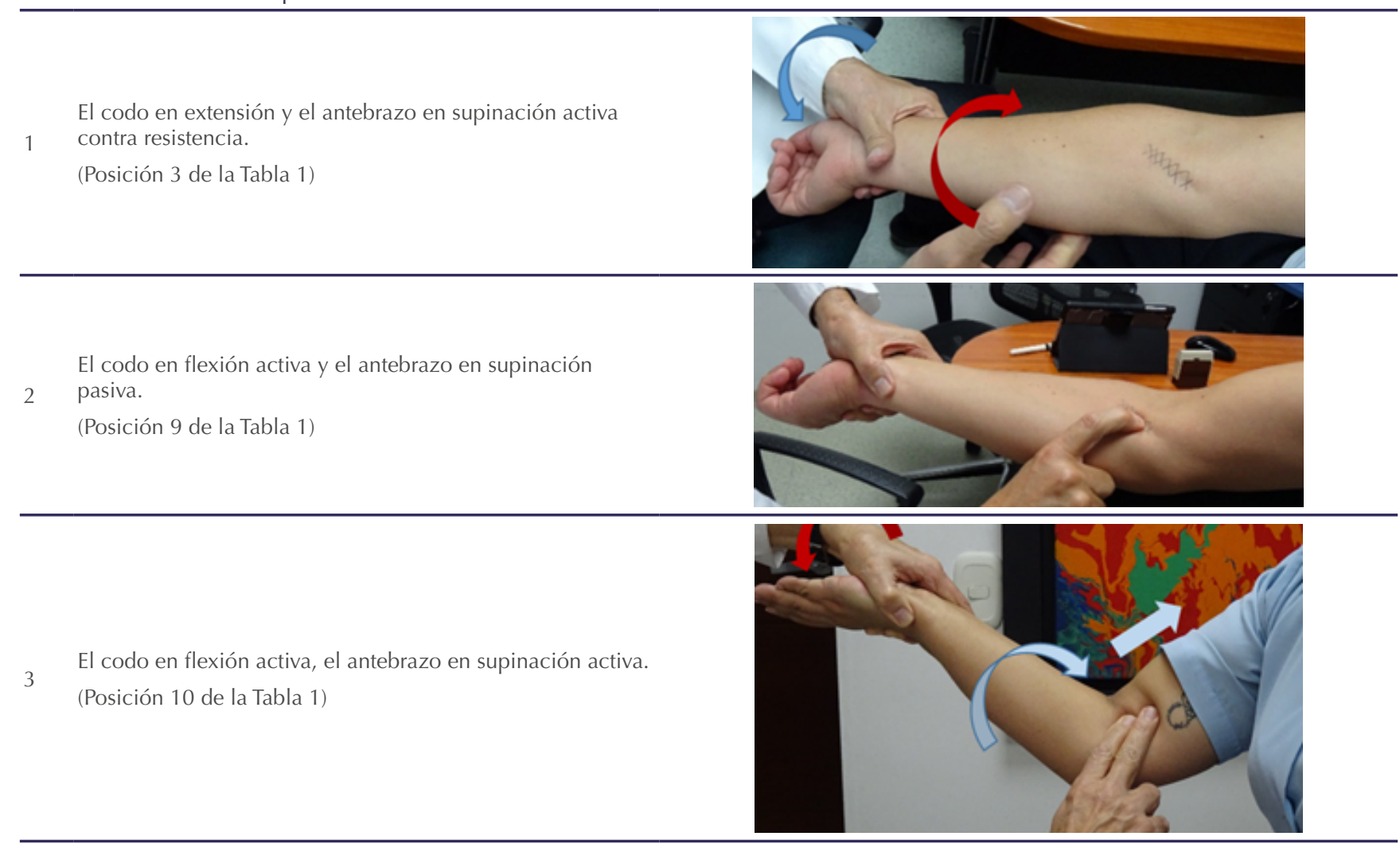

Fuente: Elaboración propia.

En los cuadros clínicos de patología aislada del lacertus fibrosus pueden no encontrarse signos de compresión del nervio mediano, ni parestesias ni disestesias, aunque en casos graves y de largo tiempo de evolución estas sí pueden estar presentes. Por lo tanto, se concluye que son dos estados patológicos diferentes: el primero, de patología primaria como un proceso inflamatorio local que podría denominarse tendinitis del lacertus fibrosus, y el segundo, de patología secundaria a la compresión que esta estructura pueda ejercer sobre los componentes vasculo-nerviosos. Es pertinente agregar que en estos casos también son negativas las maniobras para buscar otros sitios de compresión más distales.

\section{Tratamiento}

Basados en el diagnóstico de una tendinitis del lacertus fibrosus, para el tratamiento en la fase aguda se recomienda un ciclo de antiinfla- matorios no esteroideos (AINES) vía oral y la aplicación de calor local; eventualmente se puede recurrir a la aplicación de una dosis de corticoide intramuscular. Como medidas coadyuvantes se podrían considerar los recursos fisioterapéuticos, evitar actividades de fuerza mayor y realizar ejercicios de estiramiento no dolorosos, los cuales son preferibles a la inmovilización. Si estas medidas no surten efecto, se puede agregar una infiltración de corticoide $\left(1 \mathrm{o} 2 \mathrm{~cm}^{3}\right.$ de acetonida de triamcinoloma $10 \mathrm{mg} / \mathrm{mL}$ mezclado con 1 o $2 \mathrm{~cm}^{3}$ de lidocaína $1 \%$ sin epinefrina) subcutánea en forma superficial, sin penetrar el lacertus y sin que la aguja se acerque al nervio mediano. Los ejercicios de estiramiento que se indiquen, específicamente del lacertus fibrosus, deben seguir los planteamientos presentados en la Tabla 1.

En los casos crónicos o resistentes en donde no se logre mejoría después de 6 semanas está indicado el tratamiento quirúrgico para seccionar el lacertus fibrosus, el cual se puede llevar a cabo por vía abierta (Figura 10) o por vía endoscópica ${ }^{13}$.
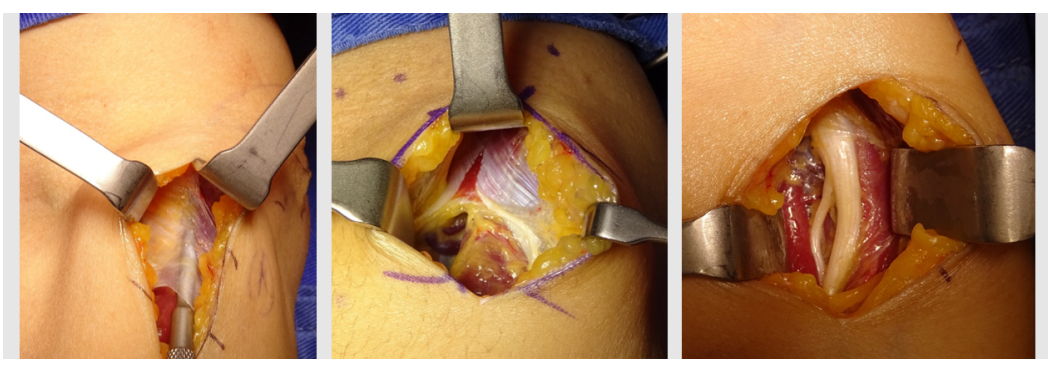

Figura 10. Liberación del lacertus fibrosus y descompresión del nervio mediano. Fuente: Documento obtenido durante la realización del estudio. 


\section{Materiales y métodos}

Se realizó una búsqueda bibliográfica de la literatura en fuentes secundarias de información a través las bases de datos PubMed, BioMed central (BMC) y Scielo, y utilizando los términos lacertus fibrosus, Pirogoff aponeurosis y bicipital aponeurosis. Se tuvieron en cuenta estudios tipo revisión y con acceso completo al texto, y no se estableció un límite de periodo de publicación, obteniendo 1 artículo en OVID, 17 artículos en PubMed, 7 artículo en BioMed Central y 1 artículo en Scielo, para un total de 26 artículos. Se tuvieron en cuenta únicamente estudios descriptivos con bases anatómicas y biomecánicas, los ensayos clínicos y los estudios que describían intervenciones quirúrgicas fueron excluídos, por lo que la muestra final estuvo constituída por 13 artículos. Se hizo una lectura de los resúmenes de estos tomando como base bibliográfica 6 de ellos.

Posteriormente, se realizó una revisión de los casos presentados entre el 13 de enero de 2015 y el 12 febrero del 2020 con diagnós- tico clínico de síndrome del lacertus fibrosus y se analizó el comportamiento epidemiológico según sus diagnósticos etiológicos y de cronicidad.

\section{Resultados}

En la revisión, se encontró que en el periodo establecido (enero de 2015 a febrero de 2020) se realizaron 4100 historias clínicas y que en 29 de estas los pacientes habían sido diagnosticados con síndrome del lacertus fibrosus: en 1 este fue el único diagnóstico; en 2 este síndrome se asoció a síndrome del pronador redondo y en 26 se encontraron simultáneamente los síndromes del lacertus fibrosus, del pronador redondo y del interóseo posterior; además, 10 de los 29 casos se asociaron al síndrome del túnel carpiano.

De igual forma, se encontró que cerca de la mitad de los casos se presentaron en pacientes de sexo femenino y en mayores de 56 años (55.1\%) (Tabla 3).

Tabla 3. Distribución de frecuencias. $(n=29)$

\begin{tabular}{c|c|c|c|ccc} 
Edad & Mujeres & $\mathbf{\%}$ & Hombres & $\mathbf{\%}$ & Total & \% \\
\hline De 26 a 35 años & 3 & 13.6 & 1 & 14.3 & 13.8 \\
\hline De 36 a 45 años & 4 & 18.2 & 1 & 14.3 & 5 & 17.2 \\
\hline De 46 a 55 años & 3 & 13.6 & 1 & 14.3 & 14.3 & 7 \\
\hline De 56 a 65 años & 6 & 27.3 & 1 & 42.9 & 9 & 24.1 \\
\hline De 66 a 75 años & 6 & 27.3 & 7 & 100.0 & 29 \\
\hline Total & 22 & 100 & 7 & 100.0 \\
\hline
\end{tabular}

Fuente: Elaboración propia.

El lado más frecuentemente afectado fue el derecho, lo cual se relacionó con la dominancia derecha en los pacientes con el diagnóstico $(65.5 \%)$; cabe resaltar que el porcentaje de afectación bilateral es menor (13.8\%) (Tabla 4).

El dolor antero-medial en el brazo distal y en el antebrazo fue el síntoma predominante en las consultas realizadas, seguido de la limitación funcional. Se tuvieron en cuenta otros síntomas como el edema y los signos de compresión del nervio mediano, los cuales no fueron reportados en dichas consultas (Tabla 5).

Finalmente, se evalúo la presencia de otros síndromes coexistentes y se encontró una baja prevalencia del síndrome del lacertus fibrosus propiamente dicho: en el $89 \%$ de los casos este síndrome estuvo asociado al síndrome del interóseo posterior y al síndrome del pronador redondo, lo que coincide con lo reportado en la literatura ${ }^{2,10}$ (Tabla 6).

Tabla 4. Relación mano dominante versus mano sintomática. ( $n=29)$.

\begin{tabular}{|c|c|c|c|c|c|c|}
\hline \multirow{2}{*}{ Lado sintomático } & \multicolumn{4}{|c|}{ Dominancia } & \multirow{2}{*}{ Total } & \multirow{2}{*}{$\%$} \\
\hline & Derecha & $\%$ & Izquierda & $\%$ & & \\
\hline Derecho & 19 & 65.5 & 1 & 3.4 & 20 & 69.0 \\
\hline Izquierdo & 5 & 17.2 & 0 & 0.0 & 5 & 17.2 \\
\hline Bilateral & 4 & 13.8 & 0 & 0.0 & 4 & 13.8 \\
\hline Total & 28 & 97 & 1 & 3.4 & 29 & 100.0 \\
\hline
\end{tabular}

Fuente: Elaboración propia.

Tabla 5. Síntomas presentados por los pacientes.

\begin{tabular}{c|cc} 
Síntomas & Frecuencia & Porcentaje \\
Dolor & 21 & $72.42 \%$ \\
\hline Edema & 8 & $27.59 \%$ \\
\hline Total & 29 & $100 \%$ \\
\hline
\end{tabular}

Fuente: Elaboración propia. 
Tabla 6. Síndromes asociados al síndrome del lacertus fibrosus. $(n=29)$.

\begin{tabular}{c|cc} 
Síndromes asociados & Frecuencia & Porcentaje \\
\hline Lacertus únicamente & 1 & $3.45 \%$ \\
\hline Lacertus + IP & 2 & $6.90 \%$ \\
\hline Lacertus + IP + PR & 26 & $89.66 \%$ \\
\hline Total & 29 & $100 \%$ \\
\hline
\end{tabular}

IP: interóseo posterior; PR: pronador redondo.

Fuente: Elaboración propia.

\section{Discusión}

La presente investigación estuvo motivada por observaciones clínicas reiterativas relacionadas con cuadros de dolor en la cara anteromedial del codo que sugerían la existencia de una patología del lacertus fibrosus.

Se hizo una revisión de la anatomía descrita de esta estructura y se precisaron aspectos de importancia, tales como la diversidad de su forma y la existencia de su capa profunda ${ }^{5,6}$. Al encontrar escasa información sobre su biomecánica ${ }^{1,2,5,7,8}$, se realizó el estudio hipotético presentado en la Tabla 1, el cual llevó a comprender que esta estructura puede ser asiento de una patología propia.

A partir de los resultados, se establece que indefectiblemente el cuadro de dolor en la cara antero-medial del codo no se puede diagnosticar únicamente bajo el concepto de síndrome del pronador redondo y se considera que la expresión "neuropatía compresiva proximal del nervio mediano", previamente sugerida ${ }^{2}$, es más apropiada y por tanto la anterior debe reservarse para las compresiones ocurridas exclusivamente en el músculo pronador.

Sin embargo, a partir de los hallazgos clínicos, se considera pertinente incorporar la expresión síndrome del lacertus fibrosus como aquí se propone, ya que este concepto ha sido de gran utilidad en la práctica clínica diaria para describir un cuadro que se puede encontrar en forma independiente o asociado con los síndromes del pronador redondo, del interóseo posterior y del túnel carpiano. Asimismo, para su diagnóstico clínico se recomienda aplicar las tres maniobras de provocación presentadas.

\section{Conclusiones}

El lacertus fibrosus es una estructura anatómica que se presenta en diversas formas anatómicas y cuyo comportamiento biomecánico es variado. Esta puede ser asiento de una patología local y dar origen a un cuadro clínico propio que podría denominarse síndrome del lacertus fibrosus. Igualmente, al lado de otras estructuras existentes alrededor del codo puede contribuir al origen de cuadros clínicos que se podrían agrupar bajo la denominación "neuropatía compresiva proximal del nervio mediano", tal como se ha sugerido con anterioridad ${ }^{2}$, aunque comúnmente se le menciona como síndrome del pronador redondo.

Con base en un estudio biomecánico hipotético, se recomienda realizar tres maniobras de provocación para definir el diagnóstico. En cuanto al tratamiento, se considera apropiado el manejo con AINES y medidas físicas para los casos recientes y el abordaje quirúrgico para los casos crónicos o de diagnóstico compuesto.

\section{Conflicto de intereses}

Ninguno declarado por los autores.

\section{Financiación}

Ninguna declarada por los autores.

\section{Agradecimientos}

Ninguno declarado por los autores.

\section{Referencias}

1. Medical Dictionary. Farlex Partner Medical Dictionary; 2012 [citado octubre 28 2020]. Lacertus fibrosus. Disponible en: https:// medical-dictionary.thefreedictionary.com/lacertus+fibrosus.

2. Caetano EB, Vieira LA, Almeida TA, Gonzales LAM, de Bona JE, Simonatto TM. Bicipital aponeurosis. Anatomical study and clinical implications. Rev Bras Ort. 2017;53(1),75-81. DOI: 10.1016/j.rboe.2017.11.014.

3. Testut L, Jacob O. Tratado de anatomía topográfica con aplicaciones medico quirúrgicas. Barcelona: Editorial Salvat; 1979.

4. Acland R. D. Acland's Video Atlas of Human Anatomy. The Upper Extremity. Vol 1. Lousiville: University of Louisville.

5. Snoeck O, Lefèvre P, Sprio E, Beslay R, Feipel V, Rooze M, Van Sint Jan S. The lacertus fibrosus of the biceps brachii muscle: an anatomical study. Surg Radiol Anat. 2014;36(7):713-9. DOI: 10.1007/s00276-013-1254-6.

6. Eames MHA, Bain GI, Fogg QA, van Riet RP. Distal biceps tendon anatomy: a cadaveric study. J Bone Joint Surg Am. 2007;89(5):1044-9. DOI: 10.2106/JBJS.D.02992.

7. Kapandji IA. Fisiologia articular: Esquemas comentados de mecanica humana. Sao Paulo: Editora Manole; 1980.

8. Recio M, Martínez V, Hernández LC, Viaño J, López-Pino MA, Jiménez M, et al. Rotura del tendón distal del bíceps. Rincón de la Imagen Archivos de Medicina del deporte. 2001;18(86):635-7.

9. Spinner M. Injuries to the Major Branches of Peripheral Nerves of the Forearm. $2^{\text {nd }}$ ed. Philadelphia: Saunders Company. .

10. Meléndez R. Cirugía de la mano. Aspectos esenciales de la práctica clínica. Bogotá D.C.: Editorial Distribuna; 2015.

11. Eversmann WW Jr. Compression and entrapment neuropathies of the upper extremity. J Hand Surg Am. 1983;8(5 Pt 2):759-66. DOI: 10.1016/s0363-5023(83)80266-4.

12. McCue FC, Alexander EJ, Baumgarten TE. Median nerve entrapment at the elbow in athletes. Operative Techniques in Sports Medicine. 1996;4(1):21-7. DOI: 10.1016/S1060-1872(96)80006-2.

13. Vergara-Amador E, Castillo-Pérez S, Tovar-Cuellar W. Liberación endoscópica del nervio mediano en el tercio proximal del antebrazo. Nuevo abordaje. Rev Esp Cir Ortop Traumatol. 2019;63(6):439-46. DOI: 10.1016/j.recot.2019.05.001. 